\title{
Surgical Treatment of Unruptured Intracranial Middle Cerebral Artery Aneurysms: Angiographic and Clinical Outcomes in 143 Aneurysms
}

\author{
Seung Won Choi, MD, Jae Sung Ahn, MD, PhD, Jung Cheol Park, MD, Do Hoon Kwon, MD, PhD, \\ Byung Duk Kwun, MD, PhD, Chang Jin Kim, MD, PhD \\ Department of Neurosurgery, Asan Medical Center, University of UIsan College of Medicine, Seoul, Korea
}

Objective : The purpose of this study was to determine the outcomes of surgical clipping in patients with unruptured middle cerebral artery (MCA) aneurysms.

Methods : A retrospective single-center database of 125 consecutive patients with 143 small MCA aneurysms $<10 \mathrm{~mm}$ ) who underwent surgical clipping was reviewed from January 2007 to December 2010. Clinical outcomes were assessed based on surgery-related complications and follow-up (mean: 17 months) using the modified Rankin scale (mRS). Angiographic outcomes were evaluated by conventional angiography ( $N=96$ ) or computed tomography angiography ( $N=29$ ) at postoperative weeks 1 and 6 .

Results : There were no cases of mortality. There were three surgery-related complications (intracranial hemorrhage, meningitis and wound infection, respectively). The hemorrhagic event caused transient neurological deficits. All patients showed good clinical outcomes during follow-up (mRS $0-1)$. There was angiographic evidence of complete occlusion in 137 aneurysms (95.8\%), a small residual neck in three aneurysms (2.2\%) and partial for three aneurysms. In the three cases with partial clipping, the decision was made preoperatively to leave the residual sac to maintain distal flow, and muscular wrapping was performed.

Conclusion : Our study demonstrates that surgical clipping of unruptured small MCA aneurysms yields favorable clinical and angiographic outcomes. Aneurysmal clipping can be safely recommended for patients with small unruptured MCA aneurysms.

Keywords Aneurysm, Middle cerebral artery, Surgical clip, Treatment outcome

\author{
$\mathrm{J}$ Cerebrovasc Endovasc Neurosurg. \\ 2012 December; 14(4):289 294 \\ Received : 10 July 2012 \\ Revised : 18 August 2012 \\ Accepted : 15 November 2012 \\ Correspondence to Jae Sung Ahn, MD, PhD \\ Department of Neurosurgery, Asan \\ Medical Center, University of Ulsan \\ College of Medicine, 88 Olympic-ro 43 gil, \\ Songpa-gu, Seoul 138-736, Korea \\ Tel : (001) 82-2-3010-3550 \\ Fax : (001) 82-2-476-6738 \\ E-mail : jsahn@amc.seoul.kr
}

This is an Open Access article distributed under the terms of the Creative Commons Attribution NonCommercial License /http://creativecommons.org/licenses/by-nc/3.0] which permits unrestricted noncommercial use distribution, and reproduction in any medium, provided the original work is properly cited.

\section{INTRODUCTION}

The number of diagnoses of unruptured intracranial aneurysms (UIAs) has increased as a result of advanced non-invasive imaging technology. As the majority of patients with UIAs are asymptomatic, the decision whether to treat UIAs or not remains debatable. ${ }^{4)}$ Although either surgical or endovascular treatment may be recommended to treat UIAs, no general consensus exists regarding which is the method of choice.

The International Study of Unruptured Intracranial Aneurysms (ISUIA) failed to arrive at a definite conclusion as to whether UIAs should be treated or not and, if so, which method should be used. ${ }^{121)}$ According to the ISUIA, ${ }^{21)}$ no treatment is recom- 
mended for asymptomatic small aneurysms located in the anterior circulation. This recommendation is based upon previous surgical outcomes compared with the natural history of such aneurysms. Furthermore, the data from the ISUIA indicate no significant difference in adverse outcomes between clipping and coiling, although endovascular coiling yields a higher rate of recanalization. Even though many studies have reported favorable outcomes following treatment of UIAs, there seems to be bias based on the treating physician: Surgery is preferred by neurosurgeons, and endovascular treatment is usually promoted by neuro-interventionists. ${ }^{25-7) 11) 13-16) 19-20) ~}$

Middle cerebral artery (MCA) aneurysms are easily accessible via surgical approach because of their relatively superficial location and configuration, but are usually not suitable for endovascular coiling. Surgical treatment thus seems to be preferred over endovascular treatment in this setting. In addition to the location and size of the aneurysm, definite risk factors for potential rupture may also exist, although this has not been shown with previous statistical analysis. ${ }^{21)}$ Hence, treating asymptomatic small aneurysms of the anterior circulation should be considered. ${ }^{19)}$

The aim of this study is to analyze the clinical and angiographic outcomes of surgical clipping of unruptured MCA aneurysms as a guide for selecting the best treatment.

\section{MATERIALS AND METHODS}

This was a retrospective study of 125 consecutive patients with 143 small MCA aneurysms (< $10 \mathrm{~mm}$ ) who underwent surgical clipping at the author's institute from January 2007 to December 2010 (135 separate operations; 125 patients; 143 MCA aneurysms surgically clipped). During this 4-year period, 52 small MCA aneurysms were treated by coil embolization.

All patients underwent conventional angiography or computed tomography (CT) angiography prior to treatment. The treatment plan was developed by a multidisciplinary team of neurosurgeons and inter- ventional neuroradiologists based on the shape and location of the aneurysm. Indication of treatment included risk factors for aneurysmal rupture such as: 1) previous aneurysmal subarachnoid hemorrhage (SAH) from a different site, 2) a family history of $\mathrm{SAH}, 3$ ) any evidence of increase in size on follow-up images, 4) presence of co-existing blebs or daughter sacs on angiography, and 5) the patients' concerns. Coil embolization was performed when there were multiple aneurysms that could not be clipped in a single operation or when patients refused to have a craniotomy. If both options were considered equal, priority was given to surgical clipping.

Postoperative conventional angiography $(\mathrm{N}=96)$ or CT angiography $(\mathrm{N}=29)$ was performed at postoperative weeks 1 and 6 to assess angiographic outcomes. All patients were followed up as outpatients, and clinical outcomes were assessed at postoperative week 6 using the modified Rankin scale (mRS). The median follow-up duration was 22.5 months (range, 3 to 62 months). A clinically favorable outcome was defined as mRS 0-1.

The statistical analysis was performed using SPSS version 16 for Windows (SPSS Inc, Chicago, IL). The Mann-Whitney test was used for analysis of continuous variables, and the Fisher's exact test and chi-square tests were used for analysis of categorical variables. We considered a value of $p=0.05$ as a level of statistical significance.

\section{RESULTS}

Patient and aneurysm characteristics are shown in Tables 1 and 2. We studied 86 women and 39 men with a mean age of 57.8 years (range, 35-75 years). Eight patients had another aneurysmal SAH. None of them had any neurological sequelae related to the previous SAH. Fifty-one (40.8\%) patients had at least one additional aneurysm, including 19 cases of bilateral MCA aneurysms. In 11 cases, ipsilateral multiple aneurysms were treated with a single stage operation. One third of the aneurysms showed com- 
Table 1. Characteristics of surgical patients

\begin{tabular}{ll}
\hline Group (N = 125) & No. of Patients (\%) \\
\hline Sex & $39(32)$ \\
Male & $86(68)$ \\
Female & 58 years $(52,64)$ \\
Age, Median (IOR) & \\
Mode of presentation & $35(2)$ \\
Incidental finding & $8(6)$ \\
Previous SAH history & $62(49)$ \\
Headache & $14(11)$ \\
Dizziness & $2(1)$ \\
Others (memory impairment, syncope) &
\end{tabular}

plicated pathology such as a combined bleb, a broad neck $(>5 \mathrm{~mm})$, or an atherosclerotic plaque within the aneurysmal neck. Thirty-five of the 135 operations were complex, utilizing other surgical measures besides clipping (e.g., wrapping or multiple clips).

Out of the 135 operations, there were no cases of mortality, but there were three cases with complications (intracranial hemorrhage, meningitis, and wound infection). No neurological deterioration associated with surgery was noted during follow-up, and all patients showed favorable clinical outcomes (mRS $0-1)$.

There was only one case of postoperative complications leading to neurological impairment during admission. A temporal lobe intracerebral hemorrhage ipsilateral to the operative site was found on a postoperative CT scan. The patient presented with transient left side hemiparesis and visual field defect. All neurological deficits resolved within two weeks. The patient was asymptomatic at the time of discharge, with the last CT scan demonstrating complete resolution of the intracerebral hemorrhage. The other two cases included postoperative wound infection and meningitis. Both patients received medical treatment, and there was no sequelae related to the infection.

Complete post-clipping obliteration was found in $95.8 \%$ of aneurysms (137 of 143 aneurysms). Three UIAs (2\%) showed minimal residual necks with postoperative conventional angiography, and incomplete partial clippings were seen in three cases $(2 \%)$; one was confirmed by CT angiography and the others by
Table 2. Aneurysm characteristics

\begin{tabular}{lc}
\hline Group ( $\mathbf{N}=143)$ & No. of Aneurysms (\%) \\
\hline Location & \\
MCA bifurcation & $121(84.6)$ \\
M1 & $18(12.5)$ \\
M2 (Distal) & $4(2.7)$ \\
Other characteristics & \\
Bleb & $39(27.2)$ \\
Atherosclerotic plaque within neck & $8(5.5)$ \\
Wide neck & $43(30)$ \\
Others & $2(1.3)$ \\
Size & \\
Median (IOR) & $4 \mathrm{~mm}(3.0,5.6)$ \\
$<5 \mathrm{~mm}$ & $89(62.2)$ \\
$5-10 \mathrm{~mm}$ & $54(37.7)$ \\
\hline
\end{tabular}

$\mathrm{MCA}=$ middle cerebral artery

Others (perforators incorporating to aneurismal neck)

conventional angiography.

Minimal residual necks remained in two of the three cases to avoid parent vessel stenosis and maintain distal flow. Severe atherosclerotic changes were found in the proximal portion of the aneurysm in both cases, and flow was diminished on intraoperative indocyanine green angiography when transient clipping was applied to the aneurysmal neck. In the third case, the size of the residual neck was too small to repair (1-1.5 mm), and no significant regrowth or change in shape appeared at follow-up. In three cases with incomplete partial clipping, a decision was made preoperatively to leave the residual sac to retain the distal flow in all cases, and muscular wrapping was performed instead.

Statistical analysis was performed to determine any correlation between adverse outcomes and the aneurysmal characteristics. The size of the aneurysm correlated with incomplete obliteration of the aneurysm post clipping $(p=0.008)$. Although other complex aneurysmal characteristics (bleb or atherosclerotic plaque within the neck) correlated with a residual neck at the clipping site, these were not statistically significant ( $p=0.39,0.36$ respectively). Clinical outcome was not found to be related with age $(p=0.34)$ and sex $(p=0.21)$ of the patients. Morbidity was not also found to be related with size $(p=0.97)$ and location $(p=0.32)$ of the aneurysms. 
Table 3. Treatment outcome of previous study for unruptured MCA aneurysms

\begin{tabular}{lllll}
\hline Study & Aneurysms, No. & Morbidity, \% & Mortality, $\%$ & Treatment modality \\
\hline Regli et al. ${ }^{19)}$ & 34 & 3 & 0 & Surgery and endovascular \\
Flamm et al. ${ }^{4)}$ & 101 & 7.5 & 0 & Surgery \\
Moroi et al. ${ }^{15)}$ & $549(201, \mathrm{MCA})$ & $2.2(2.9, \mathrm{MCA})$ & 0 & Surgery \\
Morgan et al. $^{(3)}$ & 339 & 5 & 0.3 & Surgery \\
Current study & 143 & 2 & 0 & Surgery \\
\hline
\end{tabular}

\section{DISCUSSION}

The development of non-invasive imaging technology has led to increased rates of diagnosing unruptured aneurysms. There are two main debates concerning management of unruptured aneurysms because most patients with unruptured aneurysms are asymptomatic. One is whether the aneurysm should be treated at all and the other is which treatment strategy should be chosen if it is should be surgically addressed.

According to the ISUIA, ${ }^{21)}$ the size and location of the aneurysm mainly affect the risk of rupture and no treatment is recommended for asymptomatic small (< $7 \mathrm{~mm}$ ) aneurysms located in the anterior circulation. However, there has been controversy with regard to the recommendations from the ISUIA. Based on previous studies, the Quality Assurance and Guideline Committee of the Korean Society of Cerebrovascular Surgeons recommended that even asymptomatic small $(4-7 \mathrm{~mm})$ aneurysms in patients with a life expectancy of over 10 years should be considered for treatment. ${ }^{18)}$ A recent Japanese study ${ }^{12)}$ showed that the annual rupture risk of MCA aneurysm is $0.31 \%$ and $1.56 \%$ in $5-6 \mathrm{~mm}$ and $7-9 \mathrm{~mm}$ aneurysms, respectively. However, in cases of aneurysmal rupture, the mortality rate is about $35 \%$ with moderate-to-severe disability rates being $29 \%$.

Aneurysm coiling emerged as an alternative to surgical clipping during the late 1990's. According to ISAT investigators, 1-year independent survival favors aneurysm coiling in patients with a ruptured aneurysm suitable for both treatments, although the benefit of coiling disappears at five years. ${ }^{9}$ ) They also ob- served significantly lower mortality rates at five years after coiling versus surgery. ${ }^{10)}$ Although endovascular treatment of MCA aneurysm has recently become popular with improved techniques and materials, the clinical and angiographic outcomes of coiling of MCA aneurysms have not outweighed clipping for treatment of MCA aneurysms until recently. ${ }^{25-6) 14) 19-20) ~}$ MCA aneurysms are easily accessible by various surgical approaches, and their configuration is usually not suitable for endovascular treatment. Stent-assisted or balloon remodeling coil embolization may result in more procedure-related complications (5-8\%). ${ }^{817)}$ Patient selection bias should be considered because endovascular treatment is usually performed on patients who are not surgical candidates due to poor general medical condition. However, embolization also has demonstrated merits such as shortened length of admission and fewer complications coupled with comorbidities.

It seems reasonable to design a treatment strategy based on the risk of aneurysmal rupture and the risk of adverse outcomes related to the treatment to avoid $\mathrm{SAH}$. If we can significantly reduce treatment-related morbidity and mortality, active treatment of UIAs may be reasonable. ${ }^{10)}$ In addition, when a treatment strategy is to be applied, it should take into consideration the quality of results (endovascular and neurosurgical) as well as their track record with aneurysms. Treatment outcomes should be measured with sequential quality assessment.

Studies other than the ISUIA showed similar treatment outcomes (Table 3). 3)11)13)16) In our study, only patients with small, unruptured MCA aneurysm were enrolled and treated with surgical clipping. Morbidity 
related to clipping was only seen in three cases, none of which resulted in permanent neurologic deficits. The overall clinical outcome demonstrated $100 \%$ of patients reaching favorable outcomes (mRS 0-1). Our outcomes were far superior to those of other reports. There was no case of mortality in our series. Surgical clipping of unruptured small aneurysms thus may be recommended based on our data, as opposed to leaving these untreated as shown by the ISUIA.

Our study has some limitations. First, the present study was a descriptive one with small study subjects. Second, misinterpretation may have occurred with follow-up CT angiography due to clip artifact or low resolution.

\section{CONCLUSION}

MCA aneurysms are easily accessible through various surgical approaches, and surgical clipping is considered the gold standard. We believe that surgical clipping of unruptured small MCA aneurysms results in favorable clinicoangiographic outcomes. Therefore, surgical clipping may be safely recommended for patients with small unruptured MCA aneurysms.

\section{REFERENCES}

1. International Study of Unruptured Intracranial Aneurysms Investigators. Unruptured intracranial aneurysm - risk of rupture and risks of surgical intervention. $\mathrm{N}$ Engl J Med. 1998 Dec;339(24):1725-33.

2. Brinjikji W, Lanzino G, Cloft HJ, Rabinstein A, Kallmes DF. Endovascular treatment of middle cerebral artery aneurysms: a systematic review and single-center series. Neurosurgery. 2011 Feb;68(2):397-402;discussion 402.

3. Flamm ES, Grigorian AA, Marcovici A. Multifactorial analysis of surgical outcome in patients with unruptured middle cerebral artery aneurysms. Ann Surg. 2000 Oct; 232(4):570-5.

4. Gerlach R, Beck J, Setzer M, Vatter H, Berkefeld J, Du Mesnil de Rochemont $\mathrm{R}$, et al. Treatment related morbidity of unruptured intracranial aneurysms: results of a prospective single centre series with an interdisciplinary approach over a 6 year period (1999-2005). J Neurol Neurosurg Psychiatry. 2007 Aug;78(8):864-71.

5. Guglielmi G, Vinuela F, Duckwiler G, Jahan R, Cotroneo E, Gigli R. Endovascular treatment of middle cerebral artery aneurysms. Overall perioperative results. Apropos of 113 cases. Interv Neuroradiol. 2008 Sep;14(3):241-5.

6. Iijima A, Piotin M, Mounayer C, Spelle L, Weill A, Moret J. Endovascular treatment with coils of 149 middle cerebral artery berry aneurysms. Radiology. 2005 Nov; 237(2):611-9.

7. King JT Jr, Berlin JA, Flamm ES. Morbidity and mortality from elective surgery for asymptomatic, unruptured, intracranial aneurysms: a meta-analysis. J Neurosurg. 1994 Dec;81(6):837-42.

8. Mitchell P, Kerr R, Mendelow AD, Molyneux A. Could late rebleeding overturn the superiority of cranial aneurysm coil embolization over clip ligation seen in the International Subarachnoid Aneurysm Trial? J Neurosurg. 2008 Mar;108(3):437-42.

9. Molyneux AJ, Kerr RS, Yu LM, Clarke M, Sneade M, Yarnold JA, et al. International subarachnoid aneurysm trial (ISAT) of neurosurgical clipping versus endovascular coiling in 2143 patients with ruptured intracranial aneurysms: a randomised comparison of effects on survival, dependency, seizures, rebleeding, subgroups, and aneurysm occlusion. Lancet. 2005 Sep;366(9488):809-17.

10. Molyneux AJ, Kerr RS, Birks J, Ramzi N, Yarnold J, Sneade M, et al. Risk of recurrent subarachnoid haemorrhage, death, or dependence and standardised mortality ratios after clipping or coiling of an intracranial aneurysm in the International Subarachnoid Aneurysm Trial (ISAT): long-term follow-up. Lancet Neurol. 2009 May; 8(5):427-33.

11. Morgan MK, Mahattanakul W, Davidson A, Reid J. Outcome for middle cerebral artery aneurysm surgery. Neurosurgery. 2010 Sep;67(3):755-61;discussion 761.

12. Morita A, Kirino $T$, Hashi $K$, Aoki N, Fukuhara $S$, Hashimoto N, et al. The natural course of unruptured cerebral aneurysms in a Japanese cohort. N Engl J Med. 2012 Jun;366(26):2474-82.

13. Moroi J, Hadeishi H, Suzuki A, Yasui N. Morbidity and mortality from surgical treatment of unruptured cerebral aneurysms at Research Institute for Brain and Blood Vessels-Akita. Neurosurgery. 2005 Feb;56(2):224-31.

14. Quadros RS, Gallas S, Noudel R, Rousseaux P, Pierot L. Endovascular treatment of middle cerebral artery aneurysms as first option: a single center experience of 92 aneurysms. AJNR Am J Neuroradiol. 2007 Sep;28(8):1567-72.

15. Raaymakers TW, Rinkel GJ, Limburg M, Algra A. Mortality and morbidity of surgery for unruptured intracranial aneurysms: a meta-analysis. Stroke. 1998 Aug; 29(8):1531-8.

16. Regli L, Uske A, de Tribolet N. Endovascular coil placement compared with surgical clipping for the treatment of unruptured middle cerebral artery aneurysms: a consecutive series. J Neurosurg. 1999 Jan;90(6):1025-30.

17. Ryttlefors M, Enblad P, Kerr RS, Molyneux AJ. International subarachnoid aneurysm trial of neurosurgical clipping versus endovascular coiling: subgroup analysis of 278 elderly patients. Stroke. 2008 Oct;39(10): 2720-6.

18. Seo DH, Kang HS, Kim DW, Park SQ, Song Y, Sheen $\mathrm{SH}$, et al. [Guidelines for the management of unruptured intracranial aneurysm]. Korean J Cerebrovasc Surg. 2011 Dec;13(4):279-90. Korean. 
19. Suzuki S, Tateshima S, Jahan R, Duckwiler GR, Murayama Y, Gonzalez NR, et al. Endovascular treatment of middle cerebral artery aneurysms with detachable coils: angiographic and clinical outcomes in 115 consecutive patients. Neurosurgery. 2009 May;64(5):876-88; discussion 888-9.

20. Taha MM, Nakahara I, Higashi T, Iwamuro $Y$, Iwaasa M, Watanabe $\mathrm{Y}$, et al. Endovascular embolization vs surgical clipping in treatment of cerebral aneurysms: morbidity and mortality with short-term outcome. Surg Neurol. 2006 Sep;66(3):277-84;discussion 284.

21. Wiebers DO, Whisnant JP, Huston J 3rd, Meissner I, Brown RD Jr, Piepgras DG, et al. Unruptured intracranial aneurysms: natural history, clinical outcome, and risks of surgical and endovascular treatment. Lancet. 2003 Jul;362(9378):103-10. 